\title{
"Torsade de pointes" tachycardia. Re-entry or focal activity
}

$\mathrm{Sir}$,

Naumann d'Alnoncourt et al. (Br Heart $\mathcal{F}$ 1982; 48: 213-6) have elegantly shown that the electrocardiographic appearances of torsade de pointes can be simulated by pacing from two diametrically opposed ventricular foci with appropriate continuous phasic adjustment of the two pacing rates, and conclude that a similar spontaneously occurring mechanism is a possible cause of the arrhythmia.

They agree that such appearances, however, would also be produced by: (1) a wandering focus either traversing a circuitous course or moving back and forth in a crude semicircle; and (2) a re-entry tachycardia with the exit in the two ventricles varying phasically. Another explanation is that ventricular tachycardia arises from a single fixed focus, say, in the His-Purkinje trunk, and is conducted to the left and right ventricles phasically because of varying conduction along the bundle-branches (rather like a gardener holding a hosepipe and continuously spraying it back and forth across the land).

Presumably, the phasic appearances for all these possible mechanisms would be a function of either the refractory properties of any constituent conduction or exit pathways (for example the bundle-branches) with phasic fatigue and recovery occurring, or a varying discharge frequency of the focus or foci. All of these would lead to alternating activation of two lateral poles of the heart via ramifications of the His-Purkinje network that is presumed responsible at least as the carrier and probably as the source of this arrhythmia under certain well known conditions. ${ }^{1}$

These conditions in which torsade de pointes arise may produce both abnormal excitability and conduction within the His-Purkinje system. A focal mechanism could not be distinguished from a re-entry mechanism on these grounds, nor from responses to various pharmacological or pacing interventions since all treatments affect both automaticity and conduction properties. The occurrence of bifocal ventricular extrasystoles or tachycardia in association with episodes of torsade de pointes-even if phasic fusion of their two forms could explain the latter's appearance-would not discriminate between the various mechanisms nor would a necessary association be proved.

As with so many cardiac arrhythmias, especially those arising in the ventricles, a precise knowledge of the abnormal electrophysiological mechanism causing an attack is often less important than knowledge of the most effective therapy. Though knowledge is incomplete, guidelines for the management of torsade de pointes do exist. While the authors are to be congratulated on their careful study of the electrocardiographic features of torsade de pointes, one wonders how this will affect current treatment.

\section{Reference}

1 Krikler DM, Curry PVL. Editorial: Torsade de pointes, an atypical ventricular tachycardia. $B r$ Heart $\mathcal{f} 1976$; 38: $117-$ 20

Paul VL Curry, Guy's Hospital, St Thomas Street, London SE1 9RT. 